\title{
AN IMMERSIVE VIRTUAL ENVIRONMENT FOR CONGRUENT AUDIO-VISUAL SPATIALIZED DATA SONIFICATIONS
}

\author{
Samuel Chabot and Jonas Braasch \\ Graduate Program in Architectural Acoustics \\ Rensselaer Polytechnic Institute \\ Greene Building, 110 8th St, \\ Troy, NY 12180, USA \\ chabos2@rpi.edu
}

\begin{abstract}
The use of spatialization techniques in data sonification provides system designers with an additional tool for conveying information to users. Oftentimes, spatialized data sets are meant to be experienced by a single or few users at a time. Projects at Rensselaers Collaborative-Research Augmented Immersive Virtual Environment Laboratory allow even large groups of collaborators to work within a shared virtual environment system. The lab provides an equal emphasis on the visual and audio system, with a nearly $360^{\circ}$ panoramic display and 128 -loudspeaker array housed behind the acoustically-transparent screen. The space allows for dynamic switching between immersions in recreations of physical scenes and presentations of abstract or symbolic data. Content creation for the space is not a complex process-the entire display is essentially a single desktop and straight-forward tools such as the Virtual Microphone Control allow for dynamic real-time spatialization. With the ability to target individual channels in the array, audio-visual congruency is achieved. The loudspeaker array creates a high-spatial density soundfield within which users are able to freely explore due to the virtual elimination of a so-called "sweet-spot."
\end{abstract}

\section{INTRODUCTION}

In the inherently interdisciplinary field of sonification, the universal definition is the use of non-speech audio to convey information [1]. Due to a number of key advantages presented by the human auditory system, the topic continues to expand into a largely explored field of research. These advantages include a strong sense for pattern recognition and an ability to perceive subtle and transient changes that might otherwise go overlooked or unrecognized. Additionally, humans are constantly and rapidly decoding complex auditory scenes presented by their environment [2]. Benefits such as these make sonification a powerful tool in the area of data analysis.

In recent years, multiple use cases have emerged that map qualities of a set of data to specific acoustical properties of produced sound to convey information. This method of matching characteristics of data sets to acoustical details is well-known as

(c) (i) (8) This work is licensed under Creative Commons Attribution Non Commercial 4.0 International License. The full terms of the License are available at http://creativecommons.org/licenses/by-nc/4.0 a parameter-mapping technique. Parameter-mapping is a popular framework for data sonification because it makes use of sound's multidimensionality to convey changes and trends in data. Qualities of the produced sound, such as pitch and timbre, rhythm and tempo, and loudness, to name a few, are correlated to characteristics of the data [3].

Another function of a sonification system is the ability to harness spatialization of the produced sound. By doing so, the system alters the perceived location of auditory streams, and consequently employs the human ear's capacity for attending to multiple audio cues simultaneously. Strategic positioning can strongly influence the conveyance of information and immersion within the data. There are a number of techniques to create spatialized sound, each with benefits and limitations.

\section{SPATIALIZATION TECHNIQUES}

Vector Based Amplitude Panning (VBAP) is a popular method for spatializing sound. The technique makes use of pairs or triplets of loudspeakers who's individual gains are controlled such that the perception of virtual sound sources is created within the space between [4]. The result is effective at conveying the general direction of an incoming sound source while remaining computationally tractable. The so-called sweet-spot is flexible enough that a user is not confined to one specific static location. Another favored spatialization technique involves the use of headphones to create the spatialization. Head-Related Transfer Functions (HRTFs) are convolved with the audio signal, most often provided by a general HRTF library. While an individual's own HRTF varies from person to person, the result is a fairly realistic spatialization. However, it can be computationally expensive and is limited to a single simultaneous user. The soundfield is also almost always head-locked such that head movement does not correlate to movement within the soundfield.

A powerful strategy with continuously growing interest is the Wave-Field Synthesis technique. By using a dense array of loudspeakers, artificial wavefronts from virtual sound sources can be reproduced. The signal produced at each speaker is appropriately weighted and delayed such that the synthesis of the entire array creates a discretized reproduction of the desired sound wave [5]. The prime functions of WFS are the high precision with which users perceive both the angle and distance of virtual sound sources, as well as the elimination of the sweet-spot. Unlike an HRTFs head-locked limitation, and in addition to a VBAP method allowing head movement, WFS supports multiple users moving about 
the soundfield while completely retaining an accurate rendering of the sources.

The environments in which these spatial sonifications are deployed play crucial roles in the user experience of the information. Virtual environments have become the forefront of experiential spaces. The virtualization of the world around has been discussed and developed for a number of decades-in the middle of the twentieth century, researchers were already creating what would be the fundamental basis of the virtual environments of today. Contemporary projects attempt to transport the user(s) to environments that may not otherwise be immediately accessible. An example of such a space is the Allosphere at the University of California at Santa Barbara, which incorporates a large surrounding screen and complex audio systems for the purpose of immersing the user(s) [6]. Rensselaer has recently developed such a space to enhance and build upon these foundations.

\section{COLLABORATIVE-RESEARCH AUGMENTED IMMERSIVE VIRTUAL ENVIRONMENT LABORATORY}

The Collaborative-Research Augmented Immersive Virtual Environment Laboratory (CRAIVE-Lab) is a state-of-the-art immersive virtual environment developed at Rensselaer Polytechnic Institute. The goal of this space is to support a multi-modal workspace which puts equivalent emphasis on both the auditory display and visual display. The lab is intended for use not only by a single user but a large group of users simultaneously, with a focus on both scientific and artistic works, and often the gray area in between. To accommodate a large group of people, the workspace is sized accordingly, measuring $10 \mathrm{~m}$ by $12 \mathrm{~m}$.

\subsection{Video rendering}

The space is outfitted with a $4.3 \mathrm{~m}$ tall, nearly $360^{\circ}$ projector screen surrounding the perimeter of the lab. The screen is rectangular in shape, with rounded corners between the four sides. A custom brace with C-clamps holds the screen taut smooth through the corners, shown in the right image of figure 2 .

Creating a single continuous display across the entire screen requires the use of eight high-performance projectors. To allow users the ability to approach the screen, short-throw projectors were chosen. Users can stand as close as nearly a meter in front of the screen before casting any shadow on the display. Each projector runs at a resolution of $1920 \times 1200$, creating a single cohesive and continuous desktop of $15360 \times 1200$ pixels. A Dell workstation housing two NVIDIA Quadro K5200 cards with four video outputs each allows the system to render across all eight projectors with one computer.

The eight projectors and screen were meticulously aligned using laser levels and calibrated using a software tool by Pixelwix, which provided 8000 reference points at pixel resolution. The left image of Fig. 2 shows the Pixelwix calibration grid for one calibrated corner. This program is also able to stitch the projectors into a single screen, correcting for the room's geometry and smoothly blending the individual projectors together.

The projection screen itself is made of a microperforated PVC material, rendering it acoustically transparent. This allows for sound transmission, critical because located behind the screen are 128 loudspeakers for sonification purposes.

Since the visual component is essentially a single desktop display that is already blended and corrected for the rooms geome- try, content-creation is a straight-forward process. Visualizations need only be created for the entire span of the $15 \mathrm{k}$ pixel width of the screen. For many programs such as Max/MSP/Jitter or Photoshop, this only requires defining the workspace with the correct pixel amounts. High-quality replications of real-life locations can be created using software for stitching together photographs into $360^{\circ}$ panoramas. Recently, the use of a $360^{\circ}$ recording device, the Freedom 360 GoPro rig, has allowed for the reproduction of immersive video for the lab as well. Renderings of artificial spaces and symbolic visualizations allow users to stand in environments that may otherwise not be possible to. Figure 1 shows a stitched panoramic rendering of a Roman amphitheater found in Germany.

\subsection{Audio rendering}

The laboratory is outfitted with a total of 134 loudspeakers, 128 of which are located in a horizontal array around the perimeter of the space approximately at ear-height behind the projector screen. Six of the loudspeakers are mounted from the ceiling and directed downward into the space to assist in further enhancing audio immersion. A heavy acoustic curtain, which spans the entire height and length of the screen, is hung behind the horizontal array to provide strong damping of the physical room's response. Carpeting will be installed soon over the current hard-surfaced floor to provide additional dampening.

All loudspeakers are individually routed using XLR cabling (totaling over 2 miles!) to a digital audio workstation (DAW) where they are connected in consecutive sets to 16 eight-channel preamplifiers/optical interfaces. A dedicated Mac Pro equipped with an RME HDSPe MADI FX sound card controls the audio output. The computer uses the sound card to send all 128 channels of audio for the horizontal array over two optical MADI outputs. These MADI outputs are each received by an eight-channel format converter, split into eight channels of AES-formatted output, and, subsequently, received by the preamplifiers for output to the loudspeakers. This hardware setup allows each loudspeaker channel to be accessed and targeted individually. The additional six hanging speakers are fed through a separate M-Audio 1814 interface because all of the available channels of the format converters and preamplifiers are occupied by the horizontal array. Figure 6 provides a helpful diagram, which shows the dimensions and loudspeaker channel layout of the space, courtesy of J. Carter of Rensselear. Additionally, a Sennheiser wireless microphone system with four microphone units is installed for audio input to the system. Figure 5 provides a visual of the workstations and audio interfaces that support the laboratory

The system is flexible in its production of sound, and multiple approaches can be taken. Because each channel can be addressed individually, many programs are able to simply specify which loudspeaker channel(s) to target. This allows a sound designer the ability to quickly and easily distinguish between global and local sounds. Output of auditory streams can be connected to all channels, thereby creating global sounds in the space. Likewise, these outputs may be concentrated in a single or clustered groups of channels creating local sounds in targeted regions of the lab. Combinations of these two concepts create soundfields in which the opportunity to move about the space becomes almost critical to exploring and discerning all possible information.

Because of the high density nature of the horizontal loudspeaker array, spatialization techniques that harness wave-field synthesis fundamentals are effective ways of creating soundfields 


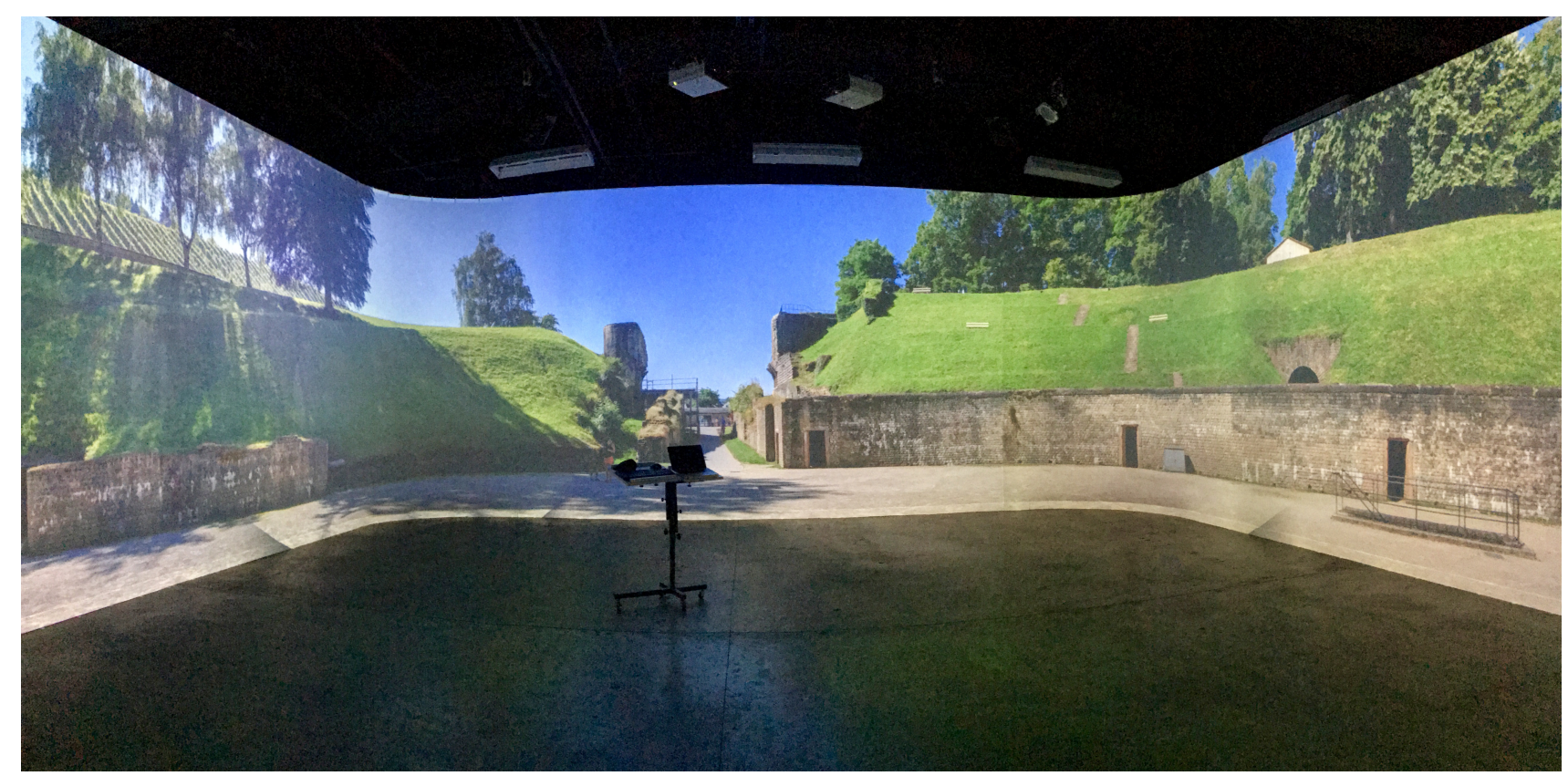

Figure 1: Panoramic rendering of Roman amphitheather in Germany

in the lab. A powerful tool developed by Jonas Braasch is the Virtual Microphone Control (ViMiC) program implemented within the Max/MSP visual coding environment. ViMiC is designed for the flexible, real-time spatialization of sound sources [7]. The program creates a computer-generated virtual space within which are placed virtual sound sources and receivers. These sound sources are user-defined audio inputs to the ViMiC module and the receivers are virtual microphones. The system calculates what would be received by the virtual microphones within the virtual space when the virtual sources radiate their sound. The appropriate pressure and delay at each microphone is considered. The received sound is then mapped back to reality through corresponding loudspeakers. The system contains many variables that the user is able to define. These include the microphone directivity patterns, sound source radiation patterns, strength of distance attenuation, and source and receiver locations and orientations within the virtual environment. Another useful ability of the Virtual Microphone Control is its implementation of the OpenSound Control protocol [8].

For use in the laboratory, the Virtual Microphone Control can be used to virtually deploy microphones around the perimeter of the workspace. Sound sources then placed within the virtual space are received by the array of virtual microphones and mapped to reality over the loudspeaker array of the CRAIVE-Lab. Fig 4 shows an example of a sound source radiating within the virtual space, and the corresponding wavefront that is reproduced at the loudspeaker array. As can also be seen in the figure, a critical benefit of sound reproduction in the CRAIVE-Lab is the elimination of the sweet-spot.

\subsection{Other systems}

To further enhance the capabilities of the CRAIVE lab, we are currently extending the CRAIVE-Lab with a smart lighting equipment

\begin{tabular}{|l||r|r|r|r|r|r|r|}
\hline $\begin{array}{l}\text { Meas. } \\
\text { Pos. }\end{array}$ & $\begin{array}{r}1 \\
\text { (L29) }\end{array}$ & $\begin{array}{r}2 \\
\text { (L30) }\end{array}$ & $\begin{array}{r}3 \\
\text { (L31) }\end{array}$ & $\begin{array}{r}4 \\
\text { (L32) }\end{array}$ & $\begin{array}{r}5 \\
\text { (L33) }\end{array}$ & $\begin{array}{r}6 \\
\text { (L34) }\end{array}$ & $\begin{array}{r}7 \\
\text { (L35) }\end{array}$ \\
\hline \hline Level & 26 & 30 & 30 & 34 & 31 & 30 & 29 \\
\hline ILD & -14.8 & -14.2 & -11.5 & -8.4 & -1.9 & 4.3 & 8.1 \\
\hline ITD & -0.50 & -0.38 & -0.27 & -0.17 & -0.02 & 0.13 & 0.23 \\
\hline
\end{tabular}

Table 1: Binaural manikin measurement data or the close measurement position for different loudspeakers ( $1 \mathrm{~m}$ distance between the binaural manikin and the front loudspeaker). From top to bottom: Relative Sound Pressure Levels in decibels, Interaural level differences in decibels, interaural time differences in milliseconds.

based on six ETC D60 LED fixtures that can vary spatially, temporally, and spectrally, depending on the content being displayed using the eight video projectors to enhance the performance of the users in the CRAIVE-Lab.

An intelligent position-tracking system estimates current user locations and head orientations as well as positioning data for other objects. For the tracking system, a hybrid visual/acoustic sensor system is being used to emulate the humans ability to extract robust information by relying simultaneously on different modalities. A network of six cameras has been installed in the CRAIVE-Lab accompanied by a 16-channel spherical ambisonic microphone with additional peripheral microphones.

\section{MEASUREMENTS}

\subsection{Methods}

To examine the spatial abilities of the space, measurements were taken in the CRAIVE-Lab for three different listener positions. All measurement positions are shown in Fig. 6. For the close distance case, a binaural manikin (Neumann K100 [9]) was placed at a 1 meter distance in front of loudspeaker 32 (L32) and the 7 loud- 


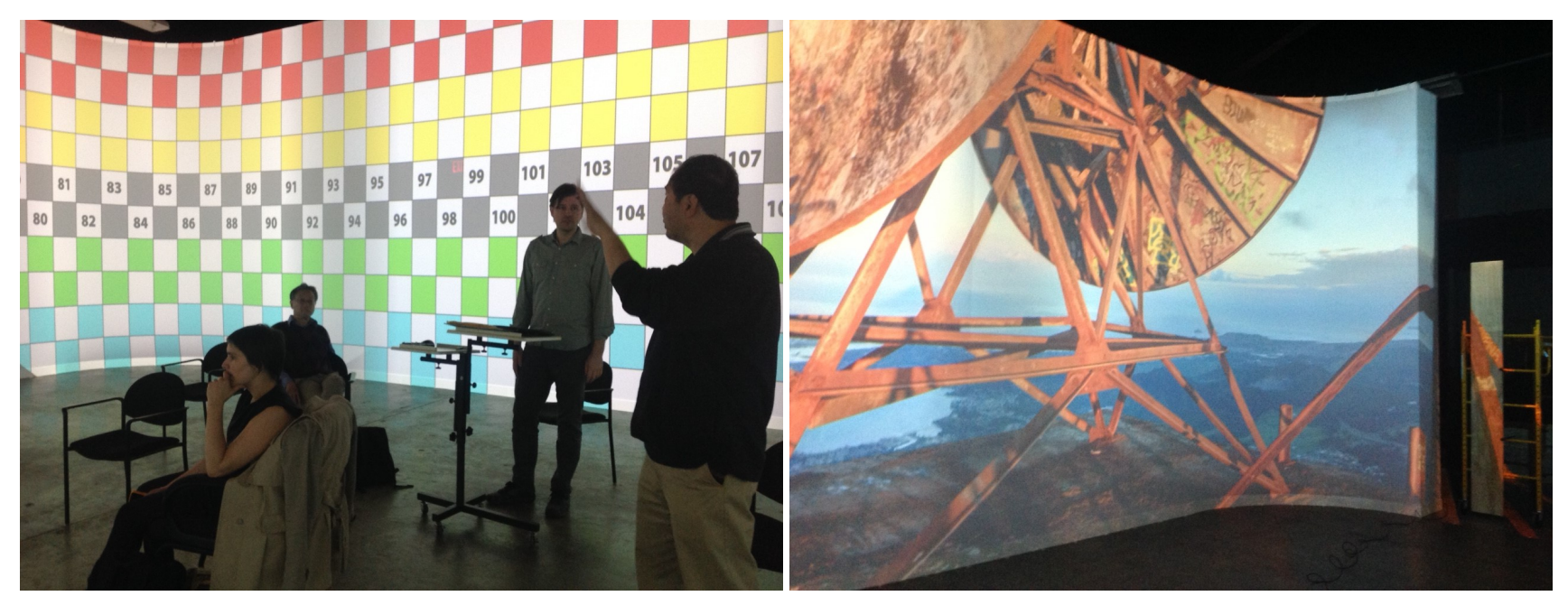

Figure 2: CRAIVE-Lab Panorama Screen. Left: Pixelwix calibration software. Right: Smooth corner solution of the CRAIVE screen.

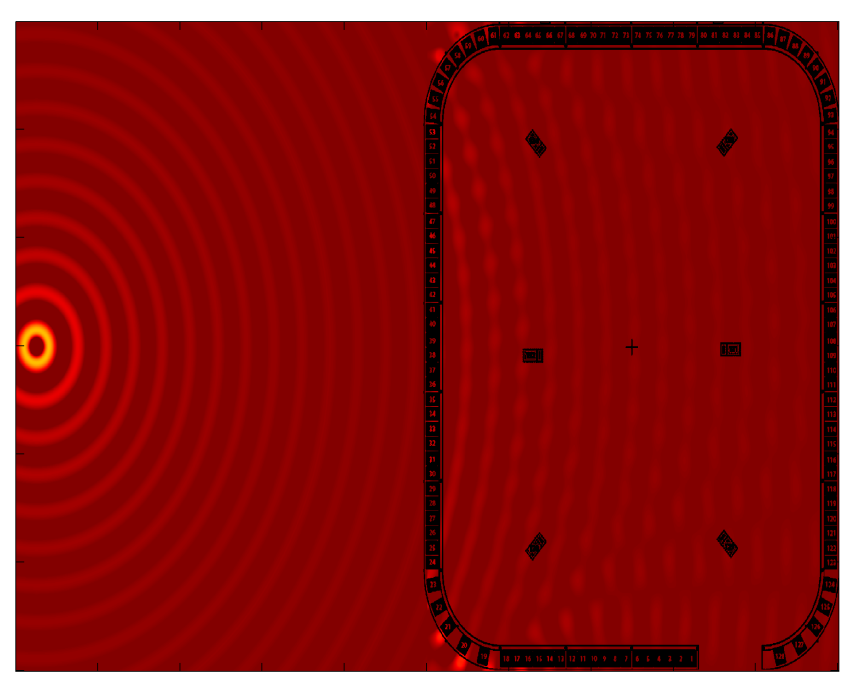

Figure 3: Synthesis of discrete loudspeakers into single wavefront for reproduction of global sound source

\begin{tabular}{|l||r|r|r|r|r|r|r|}
\hline $\begin{array}{l}\text { Meas. } \\
\text { Pos. }\end{array}$ & $\begin{array}{r}1 \\
\text { (L27) }\end{array}$ & $\begin{array}{r}2 \\
\text { (L28) }\end{array}$ & $\begin{array}{r}3 \\
\text { (L29) }\end{array}$ & $\begin{array}{r}4 \\
\text { (L30) }\end{array}$ & $\begin{array}{r}5 \\
\text { (L31) }\end{array}$ & $\begin{array}{r}6 \\
\text { (L32) }\end{array}$ & $\begin{array}{r}7 \\
\text { (L33) }\end{array}$ \\
\hline Level & 23 & 24 & 25 & 26 & 26 & 26 & 26 \\
\hline ILD & -11.6 & -10.9 & -10.3 & -9.1 & -7.2 & -4.4 & -1.3 \\
\hline ITD & -0.33 & -0.29 & -0.25 & -0.19 & -0.13 & -0.06 & 0.00 \\
\hline \hline Meas. & 8 & 9 & 10 & 11 & & & \\
Pos. & (L34) & (L35) & (L36) & (L37) & & & \\
\hline Level & 25 & 25 & 24 & 24 & & & \\
\hline ILD & 1.5 & 4.3 & 6.5 & 7.5 & & & \\
\hline ITD & 0.06 & 0.13 & 0.19 & 0.23 & & & \\
\hline
\end{tabular}

Table 2: Binaural manikin measurement data or the middle measurement position for different loudspeakers $(2.1 \mathrm{~m}$ distance between the binaural manikin and the front loudspeaker). From top to bottom: Relative Sound Pressure Levels in decibels, Interaural level differences in decibels, interaural time differences in milliseconds.

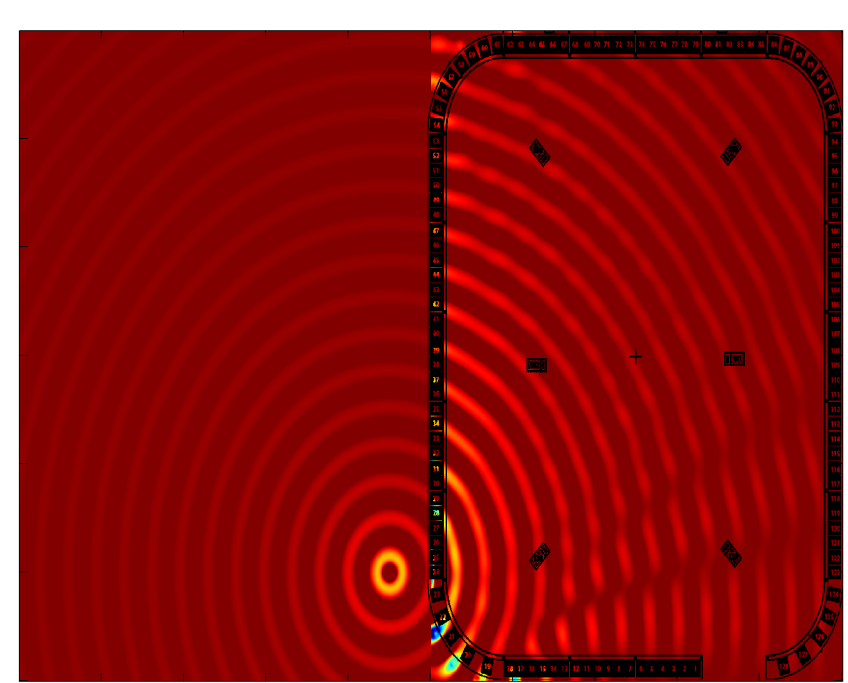

Figure 4: Synthesis of discrete loudspeakers into single wavefront for reproduction of local sound source

\begin{tabular}{|l||r|r|r|r|r|r|r|}
\hline $\begin{array}{l}\text { Meas. } \\
\text { Pos. }\end{array}$ & $\begin{array}{r}1 \\
\text { (L24) }\end{array}$ & $\begin{array}{r}2 \\
\text { (L27) }\end{array}$ & $\begin{array}{r}3 \\
\text { (L30) }\end{array}$ & $\begin{array}{r}4 \\
\text { (L33) }\end{array}$ & $\begin{array}{r}5 \\
\text { (L36) }\end{array}$ & $\begin{array}{r}6 \\
\text { (L39) }\end{array}$ & $\begin{array}{r}7 \\
\text { (L42) }\end{array}$ \\
\hline Level & 18 & 19 & 21 & 21 & 21 & 21 & 21 \\
\hline ILD & -8.3 & -9.1 & -8.4 & -7.4 & -5.6 & -2.2 & 1.3 \\
\hline ITD & -0.38 & -0.33 & -0.27 & -0.21 & -0.08 & -0.02 & 0.08 \\
\hline \hline Meas. & 8 & 9 & 10 & 11 & & & \\
Pos. & (L45) & (L48) & (L51) & (L54) & & & \\
\hline Level & 19 & 18 & 18 & 18 & & & \\
\hline ILD & 3.6 & 4.9 & 5.2 & 5.4 & & & \\
\hline ITD & 0.15 & 0.23 & 0.27 & 0.33 & & & \\
\hline
\end{tabular}

Table 3: Binaural manikin measurement data or the far measurement position for different loudspeakers ( $5 \mathrm{~m}$ distance between the binaural manikin and the front loudspeaker). From top to bottom: Relative Sound Pressure Levels in decibels, Interaural level differences in decibels, interaural time differences in milliseconds. 

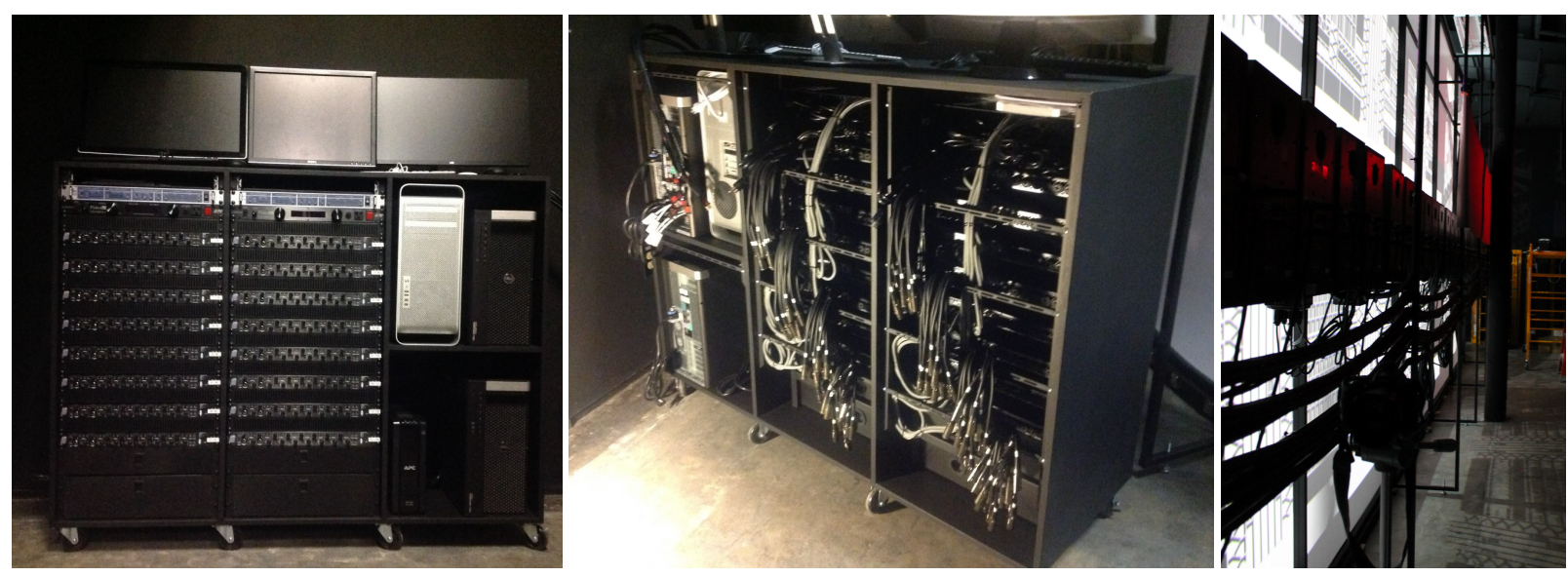

Figure 5: Left and Center: CRAIVE audio rack, right: CRAIVE cabeling.

speaker position were tested (L29-L35). For the mid position, the binaural manikin was placed at a distance of $2 \mathrm{~m}$ in front of L32 and the loudspeakers L27 to L37 were measured. For the far position, the binaural manikin was placed in the center of the CRAIVELab (5 $\mathrm{m}$ distance from the loudspeaker). A Gaussian noise burst (30 second duration) was used as the test signal, which was measured using the binaural manikin. Relative broadband sound pressure levels were calculated for each position as an average for the left and right ear signals. Interaural level differences (ILD) and interaural time differences (ITDs) were also measured for the high frequency range for ILDs $(2-20 \mathrm{kHz})$ and low frequency range for ITDs $(100-2000 \mathrm{~Hz})$.

\subsection{Results and Discussion}

As expected the loudspeaker right in front of the listener (binaural manikin) has the highest measured level for each of the three binaural-manikin positions - see Tables 1-3. Levels of $34 \mathrm{~dB}$ SPL for the close position (L32, $1 \mathrm{~m}$ distance from speaker), $26 \mathrm{~dB}$ SPL for the mid position (L32, $2 \mathrm{~m}$ distance from speaker), $21 \mathrm{~dB}$ SPL for the far position (L36, $5 \mathrm{~m}$ distance from speaker) are measured. The level reduction can be easily explained by the inverse square law.

Now to look into the approach of local vs. global acoustic viewpoints: as stated previously, this concept builds on the idea that the listener can focus much better on a local speaker to extract its auditory information, because the information to the speaker's sides will roll off much quicker than would be the case if the listener were to stand far away from the speaker. The experimental results reflect the following: at the close position, the loudspeaker levels roll off much quicker with distance from the front position than is the case for the mid or far positions. In the close position the levels of the loudspeakers 3 units away from the front speaker (L32: $34 \mathrm{~dB}$ ) roll off by approximately $6 \mathrm{~dB}$ (L29: $26 \mathrm{~dB}$ and L25: $29 \mathrm{~dB}$ ). For the mid position, the level reduction for the same speakers is only $1 \mathrm{~dB}$, and for the far position, hardly any level reduction was found when the sound was moved three speakers to the right or left (all three measurements, L30, L36 and L42 show a dB level of $21 \mathrm{~dB}$.

\subsection{Extending concepts to include wave field synthesis}

Included in the next step are the simulation of virtual sound sources to project acoustical information. Using wave field synthesis, it is possible to place a sounds at any point behind (or, with certain restriction, in front of) the screen. At a far distance, a broad wave front will be radiated using multiple speakers that does not roll off to the side - see Fig. 4. Using multiple sound sources place at different distance, local and global audio streams can be created. The global streams are placed in the background so they can be heard from any position in the lab. The local streams are presented from local loudspeakers. For the latter, the listener can move closer to these source to focus on them, while perceptually blending the other local sources out. In the center position of the lab, the listener will receive an encompassing overview of the global and local sound sources.

\section{CURRENT PROJECTS}

The CRAIVE-Lab, with it's high level of flexibility and multiple avenues for content creation and rendering, has a wide array of ongoing projects from a variety of areas. The following section will highlight three data sonifications that focus on the use the auditory display with a correlated visualization.

\subsection{Stock Market Data}

A complex set of data that is conventionally approached visually is the monitoring and analysis of current and historic stock market data. There are a variety of resources and websites tailored to graphing and visualizing this information. However, due to the multilayered complexity of the stock market, the data also lend themselves well to an acoustic approach.

The market data of the largest 128 publicly traded United States corporations are analyzed by this system. The relevant information used for the sonification includes each companys daily stock price and share volume traded. The sector of the market that each stock belongs to (e.g. financial, energy, etc.) is also included in the data set.

The sonification occurs in the visual coding environment Max/MSP. The daily stock market information is queried from an online database and loaded into dictionary objects before being 


\section{CRAIVE LAB}

audio channel layout

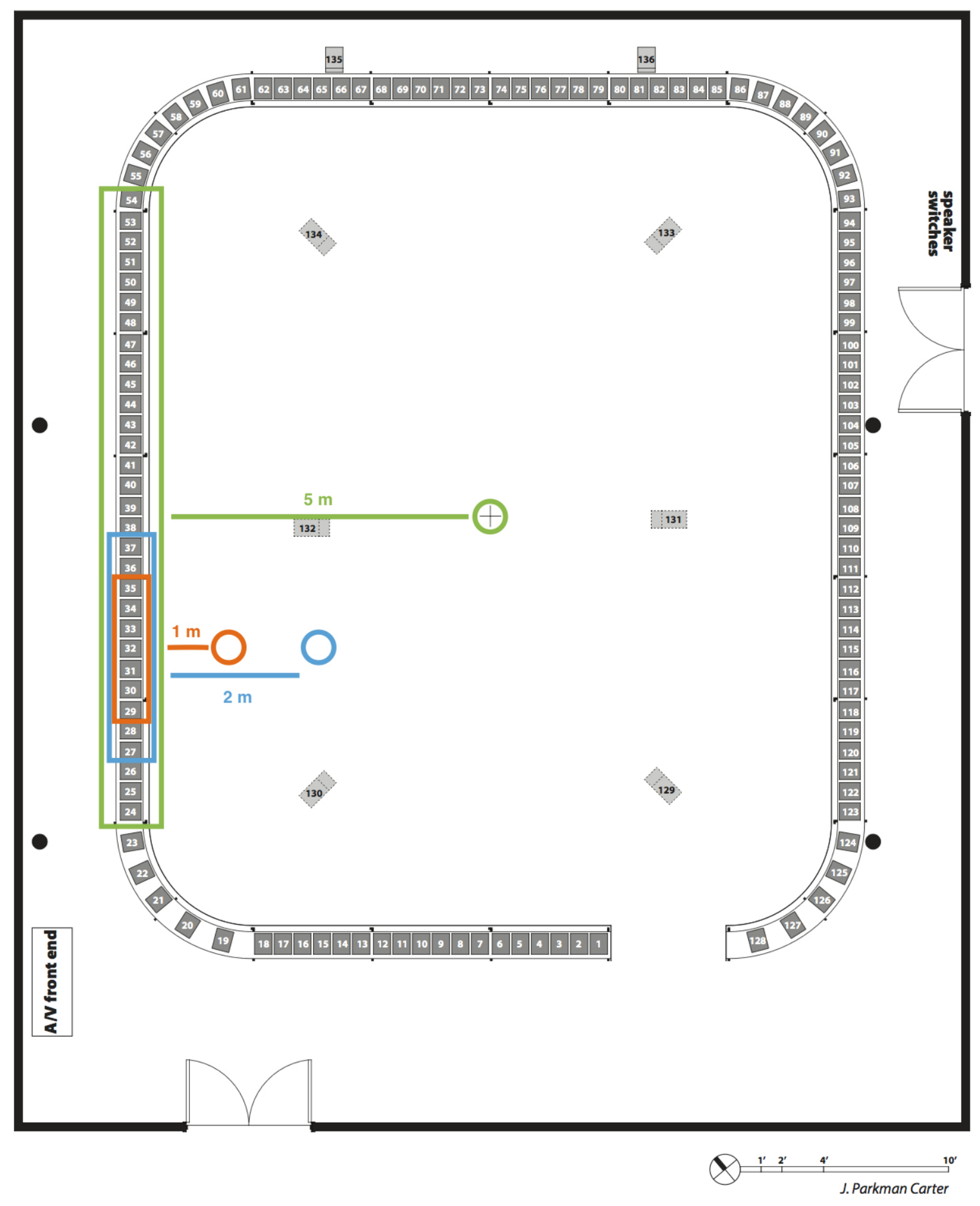

Figure 6: Graphic showing the layout and dimensions of the CRAIVE-Lab, speaker locations, and corresponding channel numbers. Also noted are measurement locations and color-coded channels utilized for each pass [10]. 


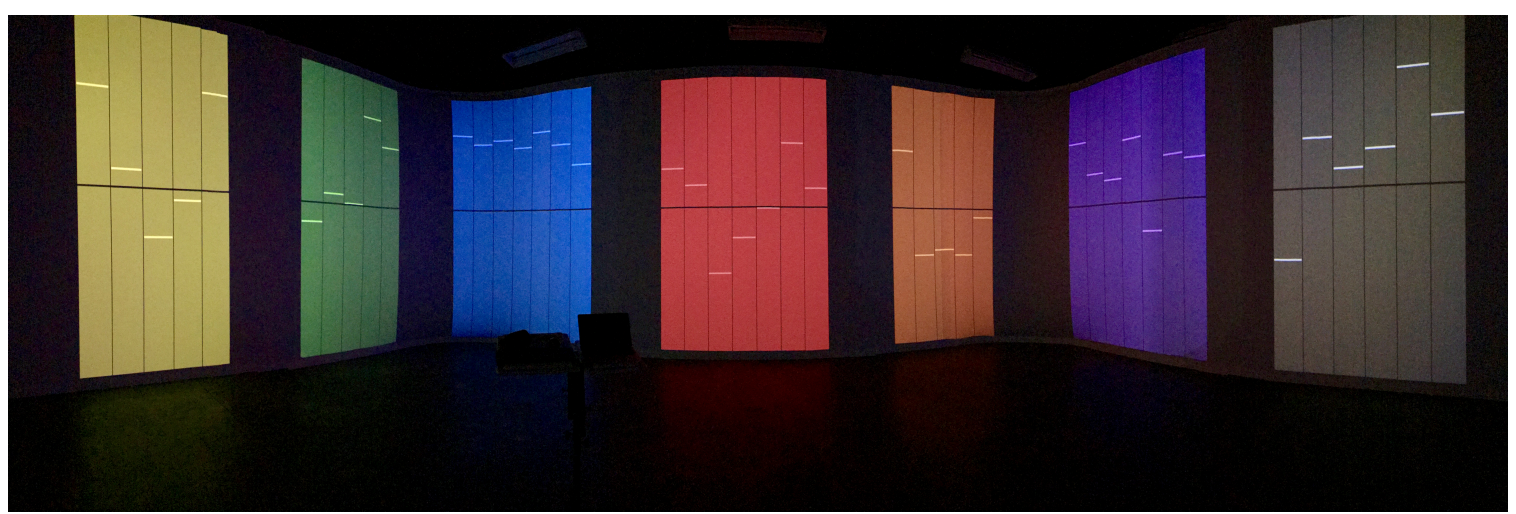

Figure 7: Visualization of stock market trends, where the top 128 publically-traded companies are clustered by sector of the market (e. g. blue-financials, green-energy, purple-technology).

processed. For each stock, the system first determines whether the price experienced a positive or negative percent change over the course of the day. This is then paired with the share volume that was traded. The share volume is mapped to the tempo of a clicktrain, where a faster tempo indicates a higher traded volume and, conversely, a slower tempo a lower traded volume. Daily volume of these 128 companies is frequently traded on the order of millions to tens of millions of shares. The volume is logarithmically scaled from a volume range of 0 to 50 million to a tempo range of 40 to 200 beats per minute, giving a higher level of granularity in the typical range of trade volume.

The composition of the click-train is determined by the percentage change in each stocks daily price. A negative percentage correlates to a pulse of white noise bursts, while a positive percentage triggers a pulse of sine tones. The pitch of the pulsing sine tone is determined by the percentage change, where a greater gain in the stocks price maps to a higher pitch sine tone. A range of 0 to 2.5 percent is scaled to a midi note range of 60 to 96 , or approximately 261 to $2093 \mathrm{~Hz}$. This process is done for all 128 stocks to create a soundscape for judging the quality and performance of the largest companies in the stock market.

Each of the generated auditory streams of the 128 corporations is assigned its own channel in the 128-loudspeaker array. The streams can be sorted into various configurations across the loudspeaker array. The configurations include ascending/descending order by total revenue as well the ability to sort by sector of the market. The system also allows for the isolation of specific sector. Figure 7 shows the generated visualization that accompanies the sonification, which contains companies clustered by their respective sectors of the market (e.g. blue denotes "financials," green denotes "energy").

Any available days of historic stock market data are usable. To isolate an interesting period of time in the market, the week of the past 2016 election, November 7 through 11, is loaded in the system. This allows the user to step through each day of the week to listen for and observe clear trends that may (and do) emerge. Clearer patterns are discernible when the corporations are sorted and clustered by sector. For example, corporations in the energy sector may be grouped in loudspeaker channels 13 through 24 , while those falling under financials may be grouped about 87 through 98. This creates a distinct separation in the spatial environment, and users can then localize trends that occur amidst a sector while comparing those trends between sectors about the environment.

\subsection{Weather Data}

Another complex data set that is ever-growing is that of meteorological data. This information is also most often approached from a visual perspective. However, as with stock market data, the multilayered nature of weather data lends itself to a sonification component. The various layers of weather data include temperature, precipitation, wind speed and direction, cloud-level, humidity, UV index. All of these also correspond to current, past, and forecasted conditions [11]

This project also utilizes the CRAIVE-Lab to present an auditory display coupled with a contextual visualization. The data itself is retrieved from the National Climate Data Center of the U.S. Department of Commerce, National Oceanic \& Atmospheric Administration (NOAA). Information regarding temperature, precipitation, and wind direction and speed collected at the Albany International Airport creates the foundation for the sonification.

The current weather conditions are used to create the sonification, which is done within Max/MSP. To replicate the current weather conditions, synthetic wind and rain is created. The wind is produced using a pink noise generator multiplied by a sine tone and sent through a resonant filter. The temperature scales the relative fundamental frequency of the wind by changing the sine tone frequency, where a higher pitch denoted a drop in temperature. The rain is created using bursts of white noise filtered to create parabolic pulses. Rumbles resembling thunder are also created with white noise run through a low-pass filter. To create a sense of realism in the audio, recordings at the location for ambient sound were also done. A spatialization of the audio is done by considering the wind direction information. Using cardinal directions mapped over the workspace, the audio stream is concentrated in corresponding loudspeakers. Users are quickly able to discern the information encoded within the sound-the temperature, wind speed and direction, and precipitation levels. This is coupled with a visualization for a complete perceptual experience.

The visualization consists of a time-lapse video taken of downtown Troy, New York in which a large unobstructed view of the sky is shown. The time-lapse was taken over the course of nearly 80 hours, with an image captured every 15 seconds. This totaled 19,142 images. A 48 hour span which saw the greatest di- 
versity of weather was exported as a 4 minute video. A small graphical visualization is overlaid on the time lapse video. This compass-like graphic is inspired by the "Wind Wheel of the program Climate Consultant. Wind direction is shown highlighted in $10^{\circ}$ increments. The color of the highlight corresponds to the relative current temperature, where dark blue denotes the coldest temperatures recorded through red corresponding to the hottest temperatures.

\subsection{EEG Data}

Currently being developed is a sonification of EEG data. This data is acquired from patients who experience epileptic seizures. There is believed to be a period of time in the minutes before the onset of a seizure in which observable patterns occur in the brain that could provide a patient with a warning of the incoming episode. An ability for providing such a warning does not currently exist because these observable patterns have not been completely identified. In assisting in recognizing these patterns, an acoustic measure is being attempted.

The EEG data is supplied in 22 separate channels, which correspond to 22 tested locations within the brain. A basic sonification abstraction charts these 22 locations to individual channels about the CRAIVE-Lab. The data is observed in low-voltage readings, which need to be mapped to an audible correlation. A parameter-mapping technique is being explored as an avenue for this data sonification. Each probe location is filtered into the appropriate brain wave frequency bands (alpha, beta, delta, theta) for a more granular analysis. The brain waves receive different timbral qualities in order to distinguish between the four. It is widely believed that indications of an incoming seizure correspond to sudden high levels of correlation between locations in the brain. The use of rhythm is being attempted for conveying these correlations by having pulses for correlated channels temporally align.

\section{CONCLUSION}

Large immersive environments such as Rensselaer's CRAIVE-Lab have the potential to alter the way people interact with each other and complex sets of data. The ability to explore and collaborate amidst a shared virtual environment at such a scale is a budding and novel approach to an exciting field of research. Providing an equal emphasis on the audio and visual components of the lab will continue to be crucial to develop congruency within a space. Environments that promote collaboration and shared physical presence will continue to enhance human interaction and comprehension of large sets of data.

\section{ACKNOWLEDGMENT}

This work is supported by the National Science Foundation (NSF \#1229391) and the Cognitive and Immersive Systems Laboratory (CISL) at Rensselaer directed Hui Su.

\section{REFERENCES}

[1] G. Kramer, B. N. Walker, T. Bonebright, P. Cook, J. H. Flowers, N. Miner, and J. Neuhoff, "Sonification report: Status of the field and research agenda," Tech. Rep.
[2] A. S. Bregman, Auditory scene analysis: the perceptual organization of sound. Cambridge, Mass.: MIT Press, 1994.

[3] T. Hermann, A. Hunt, and J. G. Neuhoff, The Sonification Handbook, Berlin, 2011.

[4] V. Pulkki, "Virtual sound source positioning using vector base amplitude panning," Journal of the Audio Engineering Society, vol. 45, no. 6, pp. 456-466, 1997.

[5] J. J. Lopez, M. Cobos, B. Pueo, and C. D. Vera, "Wave-field synthesis : State of the art and future applications," in Proceedings of the 17th International Conference on Auditory Display (ICAD2011), 2011.

[6] T. Höllerer, J. Kuchera-Morin, and X. Amatriain, "The allosphere: A large-scale immersive surround-view in- strument," IEEE Multimedia, vol. 16, no. 2, pp. 64-75, 2009.

[7] N. Peters, T. Matthews, J. Braasch, and S. Mcadams, "Spatial Sound Rendering in MAX/MSP with ViMiC," Proceedings of the International Computer Music Conference, 2008.

[8] M. Wright, A. Freed, and A. Momeni, "OpenSound Control: State of the Art 2003," Proc. NIME 2013, pp. 153-159, 2003. [Online]. Available: papers3://publication/ uuid/CEB1F875-E07B-450C-808B-0DC309595D3B

[9] Sennheiser, "Neumann Dummy Head." [Online]. Available: https://www.neumann.com/?lang=en $\{\backslash \&\} \mathrm{id}=$ current $\{\backslash$ - $\}$ microphones $\{\backslash \&\}$ cid=ku100 $\{\backslash$ - $\}$ description

[10] J. P. Carter, "Craive lab audio channel layout," 2015.

[11] L. Cunningham, "Sonfication and visualization of weather data," 2013 